\title{
Tocilizumab in Autoimmune Encephalitis Refractory to Rituximab: An Institutional Cohort Study
}

\author{
Woo-Jin Lee ${ }^{1,2}$ - Soon-Tae Lee ${ }^{1,2}$ - Jangsup Moon ${ }^{1,2}$. Jun-Sang Sunwoo ${ }^{1,2}$ • \\ Jung-Ick Byun ${ }^{1,2}$ • Jung-Ah Lim ${ }^{1,2}$ • Tae-Joon Kim ${ }^{1,2}$ • Yong-Won Shin ${ }^{1,2}$ • \\ Keon-Joo Lee ${ }^{1,2}$ - Jin-Sun Jun ${ }^{1,2} \cdot$ Han Sang Lee ${ }^{1,2} \cdot$ Soyun Kim ${ }^{1} \cdot$ Kyung-Il Park $^{1,3}$. \\ Keun-Hwa Jung ${ }^{1,2} \cdot$ Ki-Young Jung ${ }^{1,2} \cdot$ Manho Kim $^{1,2} \cdot$ Sang Kun Lee ${ }^{1,2} \cdot K_{0 n}$ Chu $^{1,2}$
}

Published online: 23 May 2016

(C) The American Society for Experimental NeuroTherapeutics, Inc. 2016 was also analyzed. The tocilizumab group showed more frequent favorable mRS scores at 2 months from treatment initiation and at the last follow-up compared with those at the relevant time points of the remaining groups. The majority $(89.5 \%)$ of the patients with clinical improvement at 1 month from tocilizumab treatment maintained a long-term favorable clinical response. No serious adverse effects of rituximab or tocilizumab were reported. Therefore, we suggest that tocilizumab might be a good treatment strategy for treating AE refractory to conventional immunotherapies and rituximab. The tocilizumab-mediated clinical improvement manifests as early at 1 month after treatment initiation.

Key Words Autoimmune disorders · encephalitis · immunotherapy $\cdot$ tocilizumab $\cdot$ prognosis

\section{Introduction}

Autoimmune encephalitis (AE) is a recently demonstrated encephalitis etiology $[1,2]$, and becoming increasingly recognized as the major cause of encephalitis [3]. The spectrum of $\mathrm{AE}$ is rapidly expanding with the continuous discovery of novel autoantibodies [4], and the clinical diagnosis of probable AE without demonstrated autoantibody $[2,5]$. The outcomes following immunotherapies are generally good, regardless of the identification of autoantibodies [6,7]. For those nonresponsive to first-line immunotherapy, second-line immunotherapies such as rituximab, which induces $\mathrm{CD} 20^{+} \mathrm{B}-$ cell destruction, yield substantial benefit [8]. However, a considerable proportion of AE might still exhibit inadequate clinical responses to rituximab and consequent unfavorable clinical outcomes $[6,8,9]$. Therefore, clinicians should consider various immune-modulation strategies for $\mathrm{AE}$ refractory to rituximab, including further maintenance of rituximab or the 
introduction of novel immunotherapies with distinct immunologic mechanisms. Extended dose of rituximab is known to potentiate its clinical response $[10,11]$. Our recent study also suggested that additional monthly rituximab could improve long-term outcomes in AE nonresponsive to first-line immunotherapies [8]. However, in treating AE that appears to be refractory to standard-dose rituximab, it is important to confirm that its further maintenance is still beneficial compared with other treatment options.

Tocilizumab is a humanized anti-interleukin (IL)-6 receptor monoclonal antibody, which blocks IL-6-mediated signal transduction [12]. Owing to the crucial role of IL-6 in stimulating both $\mathrm{B}$ and $\mathrm{T}$ cells in autoimmune processes [13-15], tocilizumab was established as efficient in various autoimmune diseases, including rheumatoid arthritis and systemic lupus erythematosus [16-18]. Among the autoimmune central nervous system (CNS) disorders, a recent study reported a powerful disease modulation by tocilizumab in rituximab-refractory neuromyelitis optica (NMO) [19-21]. Regarding AE, successful treatment of contactin-associated protein-like 2 syndrome by tocilizumab was reported [22], and some experts suggested broader use of tocilizumab in antibody-medicated encephalitis [23]. However, the efficacy of tocilizumab has not yet been evaluated in rituximab-nonresponsive $\mathrm{AE}$.

In this study, we evaluated the effect of tocilizumab compared with other treatment options in rituximab-refractory $\mathrm{AE}$ and determined indicators for a good clinical response to tocilizumab.

\section{Methods}

\section{Study Population}

From a registry of patients with a clinical suspicion of autoimmune encephalitis, 185 patients who underwent rituximab therapy between 1 May 2012 and 1 January 2015 were retrospectively reviewed. Patients were subjected to further analysis if they fulfilled the following criteria: 1) met the criteria for a clinical diagnosis of AE; 2) underwent $\geq 3$ weekly cycles of rituximab treatment as a second-line immunotherapy; 3 ) showed inadequate clinical response to rituximab therapy (defined below); and 4) were followed-up for at least 9 months [5]. Clinical diagnosis of possible $\mathrm{AE}$ was made according to the recently published guideline as follows: 1 ) subacute onset (rapid progression $<3$ months) of working memory deficits, altered mental status, or psychiatric symptoms; 2) at least 1 positive feature among new focal CNS findings, seizures not explained by a previously known seizure disorder, cerebrospinal fluid (CSF) pleocytosis, and magnetic resonance imaging (MRI) features suggestive of encephalitis (e.g., T2 hyperintensities in mediotemporal area); and 3) reasonable exclusion of alternative causes [5].
Once the diagnosis of possible AE was made, routine evaluation was performed following the recommended evaluation algorithm of encephalitis to exclude of any other possible etiologies of encephalitis, including infective, tumors, or demyelinating CNS disease (e.g., Bickerstaff 's brainstem encephalitis, acute disseminated encephalomyelitis) [5, 24]. The evaluation protocol for infectious etiologies used in our study is demonstrated in Supplemental Table 1.

Autoimmune antibodies in patients' serum/CSF were initially screened by immunohistochemistry using rat brain sections according to the method previously described [8]. Then, a cell-based immunocytochemistry method (Euroimmune Ag, Lübeck, Germany) was applied to test autoimmune-synaptic antibodies for $\mathrm{AE}$, including $N$-methyl-D-aspartate-receptor (NMDA-R), leucine-rich glioma-inactivated 1, contactinassociated protein-like 2, $\alpha$-amino-3-hydroxy-5-methyl-4isoxazolepropionic-acid-receptor 1, $\alpha$-amino-3-hydroxy-5methyl-4-isoxazolepropionic-acid-receptor 2, $\gamma$-aminobutyric acid receptor B, and amphiphysin [4]. Autoantibodies associated with classic paraneoplastic neurologic syndromes, including $\mathrm{Hu}$, Yo, $\mathrm{Ri}, \mathrm{Ma} 2, \mathrm{CV} 2 / \mathrm{CRMP} 5$, and recoverin, were also investigated using the immunoblotting method (Euroimmune $\mathrm{Ag})$ [25-27]. Once the autoantibody screening test result was positive, a definite diagnosis of $\mathrm{AE}$ was made.

For patients without demonstrated autoantibodies, intravenous aciclovir (30 mg/kg/day for 10-14 days) was empirically administered [28]. If the work-up for infectious etiologies was unrevealing, diagnosis of probable autoimmune encephalitis was made when at least 2 of the following criteria were satisfied: 1) MRI abnormalities suggestive of autoimmune encephalitis; 2) CSF pleocytosis or CSF-specific oligoclonal bands or elevated CSF IgG index; and 3) brain biopsy showing inflammatory infiltrates, excluding other disorders (e.g., tumor) [5, 8, 24].

\section{Treatments}

First-line immunotherapy included corticosteroids, intravenous immunoglobulins (IVIG), or plasmapheresis [9]. One cycle of each immunotherapy was defined as 500 to $1000 \mathrm{mg}$ methylprednisolone for 5 days, $0.4 \mathrm{~g} / \mathrm{kg}$ IVIG for 5 days, and $\geq 4$ consecutive plasmapheresis treatments. Rituximab ( $375 \mathrm{mg} / \mathrm{m}^{2}$ weekly for 4 weeks) was administrated after the determination of an inadequate clinical response to first-line immunotherapy [29]. In every patient, antihistamine and acetaminophen was administered $1 \mathrm{~h}$ before the rituximab infusion, to prevent or ameliorate infusion-related adverse effects. Clinical response to rituximab therapy was evaluated at 1 month after the last weekly rituximab session, to minimize delay in performing further treatment options, which might be fundamental in improving outcomes of AE [9]. Inadequate clinical response to rituximab was defined as lack of obvious improvement of the most disabling symptoms, based on the clinician's opinion [9]. 
For the patients fulfilling the above-mentioned criteria, 3 treatment strategies were applied based on the clinician's decision, after discussion on the efficacy, safety, and cost issues of each treatment option with the patients and caregivers. Patients were divided into 3 subgroups according to the treatment courses: 1) the group that initiated tocilizumab therapy (tocilizumab group); 2) the group that underwent monthly rituximab maintenance therapy (additional rituximab group); and 3) the group that was followed-up without any further immunotherapy (observation group).

In the tocilizumab group, tocilizumab treatment was initiated at 1 month from the last weekly rituximab cycle, with a dosage of $8 \mathrm{mg} / \mathrm{kg}$ administered for $\geq 2$ cycles in regular 1 -month intervals, which is a standard regimen used in treatment of rheumatoid arthritis or NMO [16, 17, 19, 20]. Patients who switched treatment regimens from monthly rituximab maintenance to tocilizumab owing to a lack of clinical improvement were included in the tocilizumab group. In the additional rituximab group, rituximab was administrated in 1-month intervals, beginning at 1 month after the last infusion of weekly rituximab, with the same dosage $\left(375 \mathrm{mg} / \mathrm{m}^{2}\right)$ [10].

\section{Analysis of Clinical, Laboratory, and Treatment Profiles}

Patients' medical records were evaluated, in order to obtain clinical information. Symptom profiles were analyzed according to the following categories: seizures, memory dysfunctions, language dysfunctions, behavior/psychiatric symptoms, movement disorders, autonomic dysfunction, consciousness decrement, cerebellar dysfunction, and central hypoventilation. Chest, abdomen/pelvis computed tomography, mammography, and gynecologic evaluations were performed to rule out the presence of a tumor. Whole-body positron emission tomography was performed only in select cases, when the patient was cooperative and stable. In cases where classic autoantibody was positive and investigations for a tumor were unrevealing, chest and abdomen/pelvis computed tomography were repeated at intervals of $>6$ months. Additionally, we analyzed the time lag from symptom onset to the initiation of first-line immunotherapy, rituximab, and tocilizumab administration. We also accessed the number of cycles of the first-line immunotherapy, rituximab or tocilizumab treatments.

Laboratory data, including MRI, CSF, and electroencephalography (EEG) scans obtained before the first-line immunotherapy were reviewed. In the MRI analysis, we investigated the presence of mediotemporal T2-hyperintensities, or abnormal T2-hyperintensities in any region of the cerebral hemisphere. CSF analysis included CSF lymphocyte levels, protein levels, and the presence of CSF lymphocytosis $\left(\geq 5\right.$ count $\left./ \mathrm{mm}^{3}\right)$, or protein level elevation $(\geq 40 \mathrm{mg} / \mathrm{dL})$. The EEG analysis investigated the presence of epileptic discharges in the temporal cortex. During rituximab administration, B-cell depletion was assessed using the $\mathrm{CD} 19^{+}$cell count in most patients. In the tocilizumab group, MRI/EEG/CSF data obtained before the initiation of tocilizumab therapy were also reviewed.

\section{Analysis of Safety and Outcomes}

Infusion-related adverse effects were defined as unintended and unfavorable hypersensitive reactions that developed during the infusion of rituximab or tocilizumab. Infectious adverse effects were defined as infectious complications that were considered to be consequences of those immunotherapies. Hematologic adverse events relevant to these medications were also investigated by regularly checking the complete blood count at every administration. Any adverse events were classified according to the Common Terminology Criteria for Adverse-Events v. 4.3 $[30,31]$. We described whether the noted complications resulted in the withdrawal of rituximab or tocilizumab.

As per the previous studies regarding $\mathrm{AE}$, functional status was assessed using modified Rankin Scale (mRS) scores at the initiation of first-line immunotherapy and of standard-regimen weekly rituximab, and at 1 month after the weekly rituximab administration $[6,7,9,31]$. To compare the difference of the clinical outcomes according to the treatment strategies, we set "baseline time points" defined as follows: at initiation of tocilizumab (in the tocilizumab group), at initiation of monthly rituximab administration (in the additional rituximab group), and at 1 month after the last infusion of weekly rituximab therapy (in the observation group). Additionally, we assessed the mRS score at 1 and 2 months from the baseline time points of each subgroup, as the majority of clinical responses to tocilizumab was observed within first 8 weeks of treatment, in a previous study of tocilizumab in rheumatoid arthritis and at the last follow-up ( $\geq 9$ months) [16]. Outcome parameters were designated as achievement of favorable mRS scores $(\leq 2)[6$, $7,9,31]$, and as $\mathrm{mRS}$ improvements (compared with the score at baseline time points of each subgroup) at 1 and 2 months from the baseline time points, and at the last follow-up period. To adjust the differences in baseline functional status, the parameter "favorable clinical response" was analyzed at the last follow-up period under the following definition: improvement of the mRS scores by $\geq 2$ points or achievement of the $\mathrm{mRS}$ scores $\leq 2$. Institutional cohort study for $\mathrm{AE}$ was approved by the institutional review board of Seoul National University Hospital. Written informed consent to participate was obtained from the enrolled patients or their next of kin.

\section{Statistical Analysis}

Results are presented as means $\pm \mathrm{SD}$ or $n(\%)$. Comparisons between subgroups were analyzed using an unpaired Student's $t$ test or ANOVA, followed by Tukey's b test for normally distributed variables. Categorical variables were compared using the Pearson $\chi^{2}$ test. Variables with $p$ values $<0.10$ were entered into a binary logistic regression 
analysis. SPSS 21.0 (IBM, Armonk, NY, USA) was used for all statistical analyses, and a 2-tailed $p$-value $<0.05$ was considered to indicate significance.

\section{Results}

\section{Patient Characteristics}

We initially included 185 patients in this study. According to the inclusion criteria, we excluded 5 patients with inadequate diagnostic certainty of $\mathrm{AE}, 4$ patients with inadequate treatment or follow-up, and 85 patients who showed significant clinical response to the standard-regimen rituximab therapy. Thus, the remaining 91 patients [mean age $38.8 \pm 18.5$ years (range 5-79)] were included in the final analysis and were followed-up for 21.1 \pm 9.2 months (range 9-42). Thirty patients $(33.0 \%)$ were included in the tocilizumab group, 31 $(34.0 \%)$ in the additional-rituximab group, and $30(33.0 \%)$ in the observation group (Fig. 1). According to the autoantibody tests of the patients' serum/CSF, 26 (28.6\%) patients had anti-NMDA-R antibodies, 3 (3.3\%) had anti-leucine-rich glioma-inactivated 1 antibodies, and $2(2.2 \%)$ had antiamphiphysin antibodies. The remaining $60(65.9 \%)$ patients showed negative results on the autoantibody detection test. Of the patients with anti-NMDA-R encephalitis, 6 exhibited an ovarian teratoma and underwent complete surgical resection. Except for these patients, no other patient in the study population was diagnosed with a tumor. Clinical, laboratory, and immunotherapy profiles were comparable among the subgroups (Table 1).

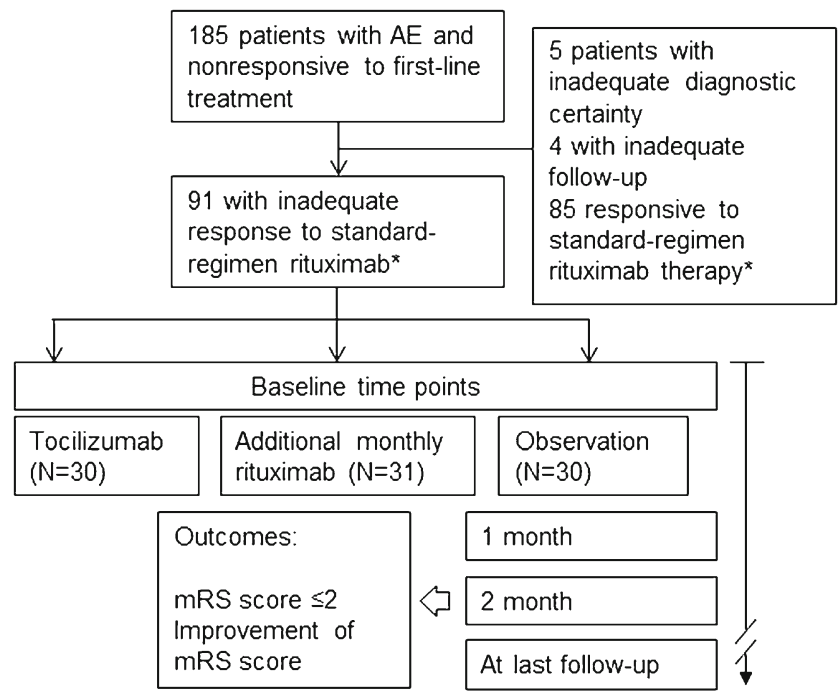

Fig. 1 Study outline *The occurrence of clinical response to the rituximab therapy was evaluated 1 month after the last weekly rituximab therapy $\mathrm{AE}=$ autoimmune encephalitis; $\mathrm{mRS}=$ modified Rankin Scale

\section{Characteristics of Immunotherapy}

First-line immunotherapy was administrated for $1.8 \pm 1.1$ cycles [median 2, interquartile range (IQR) 2-3] cycles at 14.9 \pm 20.0 weeks from the onset of symptoms. IVIG was administrated in $82(90.1 \%)$, corticosteroids in $57(62.6 \%)$, and plasmapheresis in $6(6.6 \%)$ patients. Standard-regimen rituximab was administrated for $3.9 \pm 0.3$ cycles for $22.9 \pm 23.8$ weeks from the onset of symptoms. Although not statistically significant, the observation group had a longer time to first-line immunotherapy [17.6 \pm 20.1 vs. $14.3 \pm 18.3$ (tocilizumab group), $13.3 \pm 19.5$ weeks (additional rituximab group); $p=0.281]$ and time to rituximab treatment $[27.5 \pm 25.7$ vs. $19.2 \pm 23.5$ (tocilizumab group), $21.3 \pm 12.2$ weeks (additional rituximab group); $p=0.232$ ]. In the tocilizumab group, $2.7 \pm 1.1$ cycles [median 2 , range 2-7 (IQR 2-3)] of tocilizumab therapy were administrated at $37.9 \pm 19.8$ weeks from symptom onset. Before the administration of tocilizumab, monthly rituximab was administrated for $2.1 \pm 2.4$ cycles (median 1.5, IQR $0-4$ ). In the additional rituximab group, $3.7 \pm 1.9$ cycles (median 3 , IQR $2-4$ ) of additional monthly rituximab were administrated (Table 1).

\section{Safety Profiles}

Every patient for whom $\mathrm{CD} 19^{+}$cell levels were checked $[n=79$ $(86.8 \%)$ ] during rituximab therapy achieved a near-complete depletion of CD19 $\mathrm{B}$ cells $\left(\leq 10 / \mathrm{mm}^{3}\right)$. Infusion-related adverse effects were reported in $4(4.4 \%)$ patients, all with rash (grade 1). Infectious adverse effects were reported in $7(7.7 \%)$ patients, all with pneumonia (grade 3 ) with a median onset at 28 days (range 14-56) after rituximab initiation. No complication resulted in the withdrawal of rituximab. In the tocilizumab group, 3 $(10.0 \%)$ patients reported decrement of absolute neutrophil count $(<1000 / \mathrm{dL}$; grade 3$)$. Consequently, 1 patient ceased tocilizumab treatment after the second administration cycle. No patient reported infusion-related or infectious adverse event related to tocilizumab administration. No further adverse effects associated with additional rituximab therapy were reported.

\section{Comparative Analysis of Clinical Outcomes}

The median mRS score was 4 (IQR 3-4) at the initiation of firstline immunotherapies, 4 (IQR 3-4) at the initiation of rituximab, and 3 (IQR 3-4) at 1 month after the administration of rituximab. Outcome profiles of each subgroup evaluated at every designated time point are shown in Figure 2(A-D). The proportion of patients with $\mathrm{mRS}$ scores $\leq 2$ were comparable among the subgroups at each of these time points (Table 2, Fig. 3). At the baseline time points, the median mRS score was 4 (IQR 3-4). At this time, the observation group had more frequent mRS scores $\leq 2$ as compared with the tocilizumab or additional rituximab groups ( $p=0.003$ and $p=0.012$, respectively). At 1 month from the baseline time points, $16(53.3 \%)$ patients in the 
Table 1 Intergroup comparisons of demographic, clinical, treatment, and follow-up profiles

\begin{tabular}{|c|c|c|c|c|c|}
\hline & Total $(n=91)$ & Tocilizumab $(n=30)$ & $\begin{array}{l}\text { Additional rituxiamb } \\
(n=31)\end{array}$ & Observation $(n=30)$ & $p$-value \\
\hline Male, $n(\%)$ & $43(47.3)$ & $16(53.3)$ & $13(41.9)$ & $14(46.7)$ & 0.678 \\
\hline Age of onset (years) & $38.8 \pm 18.5$ & $35.8 \pm 17.3$ & $35.4 \pm 17.8$ & $45.1 \pm 19.4$ & 0.068 \\
\hline Antibody detection, $n(\%)$ & $31(34.1)$ & $11(36.7)$ & $12(38.7)$ & $8(26.7)$ & 0.337 \\
\hline Anti-NMDA-R antibody, $n(\%)$ & $26(28.6)$ & $10(33.3)$ & $10(32.3)$ & $6(20.0)$ & 0.454 \\
\hline Tumor association, $n(\%)$ & $6(6.6)$ & $3(10.0)$ & $2(6.5)$ & $1(3.3)$ & 0.214 \\
\hline \multicolumn{6}{|l|}{ Symptom frequencies, $n(\%)$} \\
\hline Seizure & $52(57.1)$ & $20(66.7)$ & $18(58.1)$ & $14(46.7)$ & 0.299 \\
\hline Memory dysfunction & $64(70.3)$ & $21(70.0)$ & $24(77.4)$ & $19(63.3)$ & 0.493 \\
\hline Language dysfunction & $48(52.7)$ & $17(56.7)$ & $17(54.8)$ & $14(46.7)$ & 0.717 \\
\hline Behavior/psychiatric symptoms & $59(64.8)$ & $17(56.7)$ & $25(80.6)$ & $17(56.7)$ & 0.077 \\
\hline Movement disorder & $48(52.7)$ & $15(50.0)$ & $19(61.3)$ & $14(46.7)$ & 0.495 \\
\hline Autonomic dysfunction & $48(52.7)$ & $17(56.7)$ & $13(41.9)$ & $18(60.0)$ & 0.329 \\
\hline Consciousness decrement & $47(51.6)$ & $15(50.0)$ & $18(58.1)$ & $14(46.7)$ & 0.664 \\
\hline Cerebellar dysfunction & $13(14.3)$ & $2(6.7)$ & $4(12.9)$ & $7(23.3)$ & 0.180 \\
\hline Central hypoventilation & $20(22.0)$ & $8(26.7)$ & $7(22.6)$ & $5(16.7)$ & 0.651 \\
\hline \multicolumn{6}{|l|}{ CSF/MRI/EEG profiles } \\
\hline CSF lymphocyte level & $22.1 \pm 66.0$ & $33.6 \pm 83.1$ & $22.2 \pm 73.3$ & $10.5 \pm 28.0$ & 0.403 \\
\hline CSF protein level & $49.4 \pm 33.7$ & $53.6 \pm 35.6$ & $51.6 \pm 41.2$ & $42.8 \pm 20.9$ & 0.419 \\
\hline T2 abnormal HSI, $n(\%)$ & $13(14.3)$ & $4(13.3)$ & $3(9.7)$ & $6(20.0)$ & 0.516 \\
\hline Medial temporal T2 HSI, $n(\%)$ & $55(60.4)$ & $17(56.7)$ & $21(67.7)$ & $17(56.7)$ & 0.601 \\
\hline EEG epileptic discharges, $n(\%)$ & $44(48.4)$ & $16(53.3)$ & $16(51.6)$ & $12(40.0)$ & 0.632 \\
\hline Time to first-line immunotherapy (weeks) & $14.9 \pm 20.0$ & $14.3 \pm 18.3$ & $13.3 \pm 19.5$ & $17.6 \pm 20.1$ & 0.281 \\
\hline Total cycle of first-line immunotherapy & $\begin{array}{l}1.8 \pm 1.1 \\
2(1-2)\end{array}$ & $\begin{array}{l}1.8 \pm 0.7 \\
2(1-2)\end{array}$ & $\begin{array}{l}1.7 \pm 0.8 \\
2(1-2)\end{array}$ & $\begin{array}{l}1.8 \pm 1.7 \\
1(1-2)\end{array}$ & 0.938 \\
\hline Time to rituximab administration (weeks) & $22.9 \pm 23.8$ & $19.2 \pm 23.5$ & $21.3 \pm 12.2$ & $27.5 \pm 25.7$ & 0.232 \\
\hline Weekly rituximab cycle & $\begin{array}{l}3.9 \pm 0.3 \\
4(4-4)\end{array}$ & $\begin{array}{l}3.9 \pm 0.3 \\
4(4-4)\end{array}$ & $\begin{array}{l}4.0 \pm 0.2 \\
4(4-4)\end{array}$ & $\begin{array}{l}3.9 \pm 0.3 \\
4(4-4)\end{array}$ & 0.059 \\
\hline Additional monthly rituximab cycle & $\begin{array}{l}2.9 \pm 2.2 \\
2(0-3)\end{array}$ & $\begin{array}{l}2.1 \pm 2.4 \\
1.5(0-4)\end{array}$ & $\begin{array}{l}3.7 \pm 1.9 \\
3(2-4)\end{array}$ & - & $0.004 *$ \\
\hline Follow-up duration (months) & $21.1 \pm 9.2$ & $19.9 \pm 9.4$ & $20.2 \pm 9.2$ & $23.4 \pm 9.0$ & 0.273 \\
\hline
\end{tabular}

Data are reported as mean $\pm \mathrm{SD}$ or as median (interquartile range) unless otherwise indicated

NMDA-R $=N$-methyl-D-aspartate receptor; $\mathrm{CSF}=$ cerebrospinal fluid $\mathrm{MRI}=$ magnetic resonance image $; \mathrm{EEG}=$ electroencephalography his $=$ high signal intensity lesion

$* p<0.05$

tocilizumab group had $\mathrm{mRS}$ scores $\leq 2$, which was significantly more frequent than the additional rituximab group $[n=4$ $(12.9 \%) ; p=0.002]$. At 2 months from the baseline time points, the tocilizumab group showed a significantly higher frequency of $\mathrm{mRS} \leq 2[n=18(60.0 \%)]$ compared with the additional rituximab group $[n=6(19.4 \%) ; p=0.002]$ and the observation group $[n=8(26.7 \%) ; p=0.014]$. At the last follow-up, the frequency of $\mathrm{mRS} \leq 2$ in the tocilizumab group $[n=18$ $(60.0 \%)$ ] remained significantly higher than in the additional rituximab group $[n=7(22.6 \%) ; p=0.006]$ and the observation group $[n=8(26.7 \%) ; p=0.016]$. The tocilizumab group also showed higher frequencies of mRS improvement at every designated time point $(p<0.001)$, and favorable clinical responses $(p<0.001)$ compared with the other groups (Table 2, Fig. 3$)$.

\section{Predictors for a Favorable Clinical Response to Tocilizumab}

In univariate analysis, none of the clinical or laboratory variables showed significant association with a favorable clinical response to tocilizumab (Supplemental Table 2). However, most of the patients with clinical improvements at the early treatment phase maintained a long-term favorable clinical response to tocilizumab. Among the 19 patients who showed mRS improvement at 1 month from tocilizumab initiation, 17 $(89.5 \%)$ had a favorable clinical response at their last followup, whereas only $1(9.1 \%)$ of the 11 patients without mRS improvement at 1 month achieved a favorable clinical response $(p<0.001)$. 


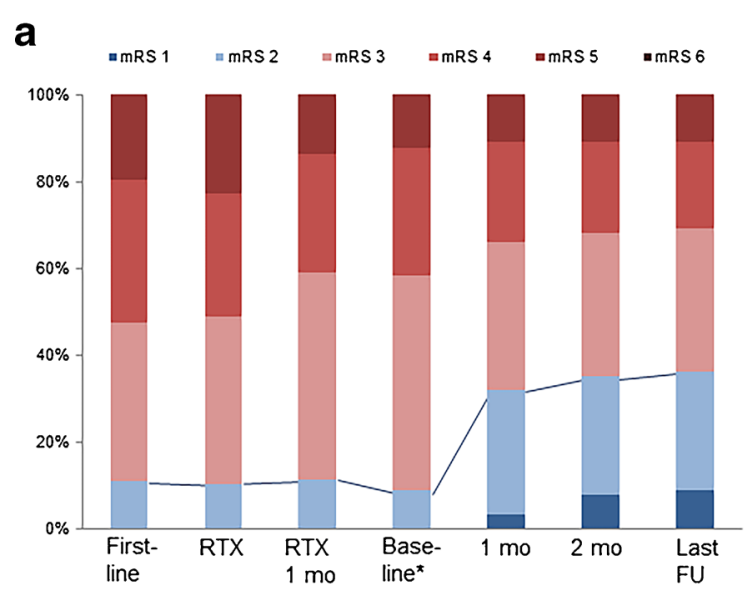

C

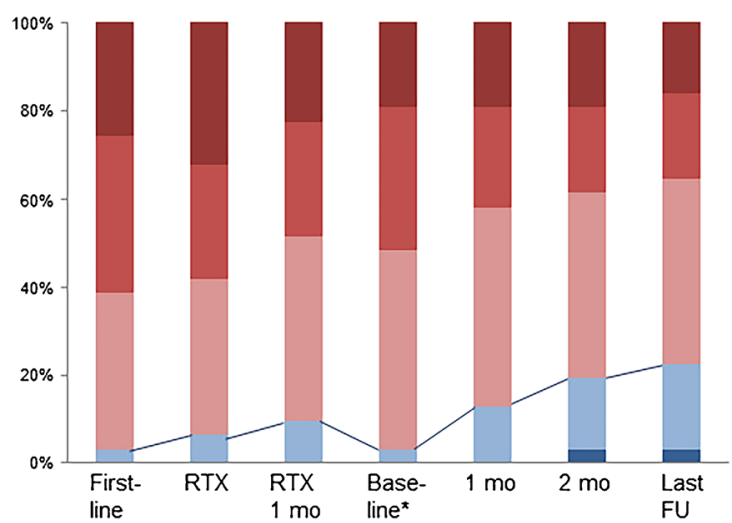

Fig. 2 Modified Rankin Scale (mRS) score profiles during the courses of immunotherapy. mRS scores are compared across (A) all patients and (BD) subgroups. The groups are as follows: (A) all patients $(n=91)$, (B) the group with tocilizumab therapy $(n=30),(\mathrm{C})$ the group with additional monthly rituximab maintenance $(n=31)$, and (D) the group with followup without further immunotherapy $(n=30)$. The solid lines represent the change in the frequencies of mRS scores 0 to 2 at each time point during the immunotherapy *Baseline: at the time of tocilizumab initiation in the

\section{Discussion}

This study revealed that the clinical benefit of tocilizumab treatment in AE refractory to the first-line immunotherapy and standard-regimen rituximab was statistically significant over observation without further immunotherapies and maintenance of monthly rituximab. Furthermore, most of the responders to tocilizumab showed clinical improvements during the early treatment phase. In addition, tocilizumab showed a tolerable safety profile in AE. Some preceding studies had revealed the efficacy of tocilizumab in rituximab-refractory NMO [19-21], and a case report highlighted successful treatment of AE with tocilizumab [22]. However, to our knowledge, this is the first study to demonstrate the clinical efficacy of tocilizumab in rituximab-refractory AE.

The efficacy of tocilizumab can be explained by the extensive pathophysiologic mechanisms by which IL-6 induces and enhances autoimmunity [13-15, 32-34].

\section{b}

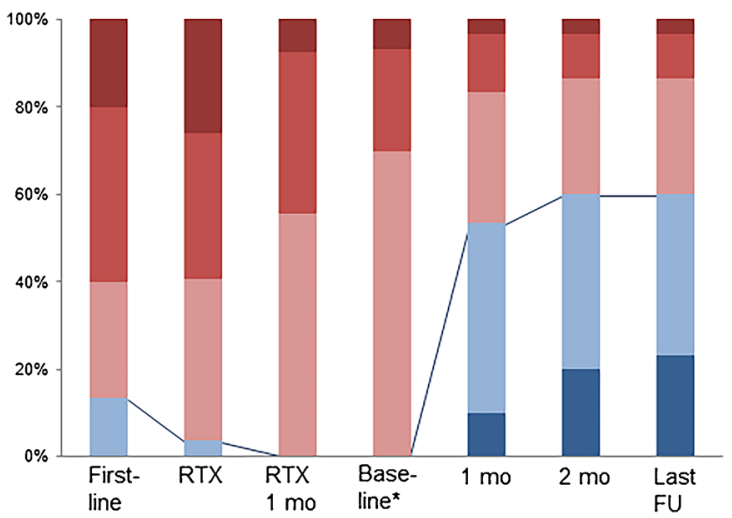

d

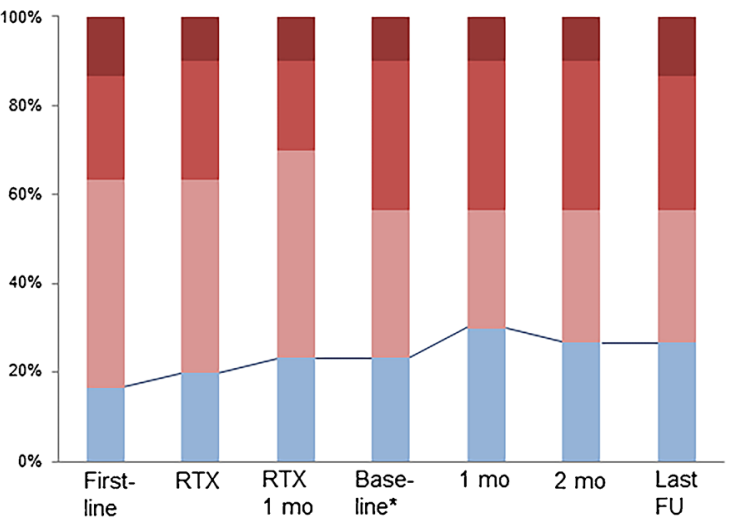

tocilizumab group, at the time of monthly rituximab therapy initiation in the additional rituximab group, and at 1 month after the last cycle of weekly rituximab therapy in the observation group First-line $=$ at initiation of first-line immunotherapies; RTX $=$ at initiation of standardregimen weekly rituximab; RTX $1 \mathrm{mo}=$ at 1 month from the initiation of weekly rituximab therapy; $1 \mathrm{mo}=$ at 1 month from the baseline time points; $2 \mathrm{mo}=$ at 2 months from the baseline time points; last $\mathrm{FU}=$ at the last clinical follow-up point

IL-6 not only induces B-cell differentiation and proliferation $[12,15]$, but also promotes the differentiation of a main inducer of autoimmune tissue damage, IL-17-producing T-helper cells, from naive $\mathrm{T}$ cells [32]. IL-6 inhibits regulatory T-cell differentiation, which is crucial in maintaining the balance against IL-17-producing T-helper cells in regulating autoimmune processes [14]. Furthermore, IL-6 stimulates the differentiation of $\mathrm{CD} 8^{+}$cytotoxic $\mathrm{T}$ cells, which promote excitotoxicity-induced neuronal damage $[12,13]$. Considering that rituximab does not affect cytokines or antibody-producing plasma cells, we suggest that tocilizumab could potentially represent a good strategy for $\mathrm{AE}$ with inadequate response to rituximab, by blocking IL-6 and thereby ameliorating autoimmune neuronal damage with multidirectional mechanisms.

In this study, we evaluated the rituximab responsiveness 1 month after the standard rituximab treatment, based 
Table 2 Intergroup comparisons of outcome profiles

\begin{tabular}{|c|c|c|c|c|c|c|}
\hline & $\begin{array}{l}\text { Total } \\
(n=91)\end{array}$ & $\begin{array}{l}\text { Tocilizumab } \\
(n=30)\end{array}$ & $\begin{array}{l}\text { Additional } \\
\text { rituxiamb }(n=31)\end{array}$ & $\begin{array}{l}\text { Observation } \\
(n=30)\end{array}$ & $p$-value & Tukey's b \\
\hline $\mathrm{mRS}$ at first-line immunotherapy & $4(3-4)$ & $4(3-4)$ & $4(3-5)$ & $3(3-4)$ & - & \\
\hline $\mathrm{mRS} \leq 2$ at first-line immunotherapy, $n(\%)$ & $10(11.0)$ & $4(13.3)$ & $1(3.2)$ & $5(16.7)$ & 0.221 & \\
\hline $\mathrm{mRS}$ at rituximab initiation & $4(3-4)$ & $4(3-5)$ & $4(3-5)$ & $3(3-4)$ & - & \\
\hline $\mathrm{mRS} \leq 2$ at rituximab initiation, $n(\%)$ & $12(13.2)$ & $4(13.3)$ & $2(6.5)$ & $6(20.0)$ & 0.302 & \\
\hline $\mathrm{mRS}$ at 1 month from rituximab & $3(3-4)$ & $3(3-4)$ & $3(3-4)$ & $3(2.75-4.00)$ & - & \\
\hline $\mathrm{mRS} \leq 2$ at 1 month from rituximab, $n(\%)$ & $10(11.0)$ & $0(0.0)$ & $3(9.7)$ & $7(23.3)$ & 0.230 & \\
\hline $\mathrm{mRS}$ at baseline time points* & $3(3-4)$ & $3(3-4)$ & $4(3-4)$ & $3(2.75-4.00)$ & - & \\
\hline $\mathrm{mRS} \geq 4$ at baseline time points, $n(\%)$ & $38(41.8)$ & $9(30.0)$ & $16(51.6)$ & $13(43.3)$ & 0.232 & \\
\hline $\mathrm{mRS} \leq 2$ at baseline time points, $n(\%)$ & $8(8.8)$ & $0(0.0)$ & $1(3.2)$ & $7(23.3)$ & 0.002 & $0.003^{\dagger}, 0.012^{\dagger}$ \\
\hline $\mathrm{mRS}$ at 1 month from baseline time points* & $3(2-4)$ & $2(2-3)$ & $3(3-4)$ & $3(2-4)$ & - & \\
\hline $\mathrm{mRS} \leq 2$ at 1 month from baseline time points, $n(\%)$ & $29(31.9)$ & $16(53.3)$ & $4(12.9)$ & $9(30.0)$ & 0.003 & $0.002^{\S}$ \\
\hline $\begin{array}{l}\text { mRS improvement at } 1 \text { month from baseline time } \\
\text { points, } n(\%)\end{array}$ & $26(28.6)$ & $19(63.3)$ & $5(16.1)$ & $2(6.7)$ & $<0.001$ & $<0.001^{\dagger, \S}$ \\
\hline $\mathrm{mRS}$ at 2 months from baseline time points* & $3(2-4)$ & $2(2-3)$ & $3(3-4)$ & $3(2-4)$ & - & \\
\hline $\mathrm{mRS} \leq 2$ at 2 months from baseline time points, $n(\%)$ & $32(35.2)$ & $18(60.0)$ & $6(19.4)$ & $8(26.7)$ & 0.002 & $0.014^{\dagger}, 0.002^{\S}$ \\
\hline $\begin{array}{l}\text { mRS improvement at } 2 \text { months from baseline time } \\
\quad \text { points, } n(\%)\end{array}$ & $30(33.0)$ & $21(70.0)$ & $7(22.6)$ & $2(6.7)$ & $<0.001$ & $<0.001^{\dagger, \S}$ \\
\hline $\mathrm{mRS}$ at last follow-up & $3(2-4)$ & $2(1.75-3.00)$ & $3(3-4)$ & $3(2-4)$ & - & \\
\hline $\mathrm{mRS} \leq 2$ at last follow-up, $n(\%)$ & $33(36.3)$ & $18(60.0)$ & $7(22.6)$ & $8(26.7)$ & 0.003 & $0.016^{\dagger}, 0.006^{\S}$ \\
\hline mRS improvement at last follow-up, $n(\%)$ & $32(35.2)$ & $21(70.0)$ & $9(29.0)$ & $2(6.7)$ & $<0.001$ & $<0.001^{\dagger, \S}$ \\
\hline Favorable clinical response at last follow-up, $n(\%)$ & $26(28.6)$ & $18(60.0)$ & $6(19.4)$ & $2(6.7)$ & $<0.001$ & $<0.001^{\dagger}$, \\
\hline
\end{tabular}

Data are reported as median (interquartile range) unless otherwise indicated

*Corresponds to mRS at the time of tocilizumab initiation in the tocilizumab group, at initiation of additional rituximab for additional rituximab group, and at 1 month from the last rituximab therapy for observation group, respectively

mRS: modified Rankin Scale, IQR: interquartile range,

${ }^{\dagger} p<0.05$ for tocilizumab group $v s$ observation group

${ }^{\star} p<0.05$ for additional rituximab group $v$ s observation group

$\S_{p}<0.05$ for tocilizumab group $v s$ additional rituximab group

on the fact that timely control of disease is essential to improve outcomes in AE [35], and that early discrimination of drug responsiveness is fundamental in the considering further treatment options. In this study, clinical improvement from standard-regimen rituximab was reported in $85 / 176(48.3 \%)$ patients [29/55 $(52.7 \%)$ for the antiNMDA-R encephalitis subgroup]. This is relatively lower than that found in a cohort of patients with anti-NMDA-R encephalitis, which reported achievement of 24-month $m R S \leq 2$ with second-line immunotherapy in $84 / 125$ patients $(67.2 \%)$ [9]. This might suggest the possibility of a delayed clinical improvement after rituximab, or might reflect the slowly improving natural course of AE. However, the clinical status of the observation group did not improve throughout the follow-up. Considering that rituximab rapidly depletes the population of $\mathrm{CD}_{1} 9^{+} \mathrm{B}$ cells within 3 weeks [36], the delayed improvements might have occurred in the immunotherapy-responsive group and not in the rituximab-refractory group.
Therefore, we think that discrimination of rituximab responsiveness at 1 month might be a pragmatic time for deciding whether to perform tocilizumab treatment. We have previously reported that additional monthly rituximab can potentiate the clinical effect of rituximab in $\mathrm{AE}$ [8]. However, in this study, only a small proportion $[n=6$ $(19.4 \%)$ ] of the nonresponders to standard weekly rituximab therapy showed clinical response to additional monthly rituximab.

Compared with a previous study regarding NMO, the cycles of tocilizumab treatment were fewer, and the duration of follow-up was shorter in this study [21]. However, in contrast to $\mathrm{NMO}$, which has a $60 \%$ rate of recurrence during 12 months [37], AE is a relatively monophasic disease with a low frequency (approximately $20 \%$ ) of relapse [4]. Additionally in this study, the high frequency of $\mathrm{mRS} \leq 2$ at 1 month from tocilizumab initiation was maintained throughout the rest of the follow-up. Therefore, in AE, fewer cycles of tocilizumab might substantively 


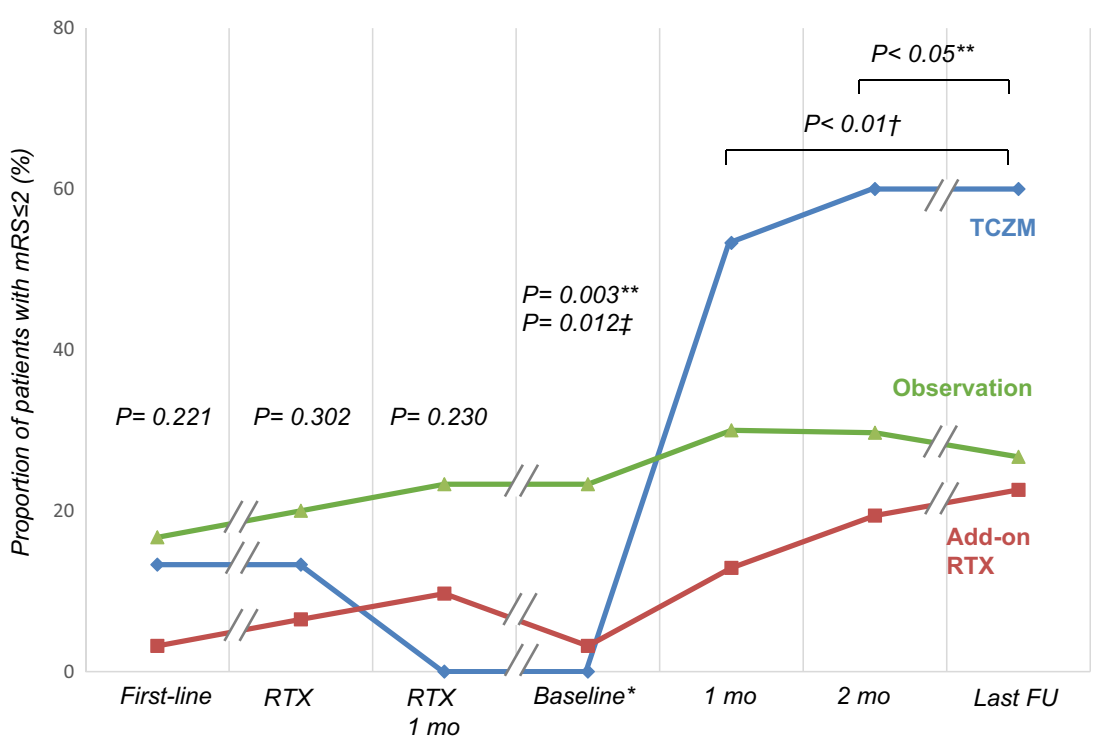

Fig. 3 Intergroup comparisons of the proportion of patients with favorable modified Rankin Scale scores (mRS) during the courses of immunotherapy. Each time point during the courses of immunotherapy were denoted as follows: first-line $=$ at initiation of first-line immunotherapies; RTX $=$ at initiation of standard-regimen weekly rituximab; RTX $1 \mathrm{mo}=$ at 1 month from the initiation of weekly rituximab therapy; $1 \mathrm{mo}=$ at 1 month from the baseline time points; 2 $\mathrm{mo}=$ at 2 months from the baseline time points; last $\mathrm{FU}=$ at the last

improve the long-term outcomes, and the clinical response to tocilizumab treatment can be detected at early phase and be maintained throughout long-term follow-up.

We found that the frequency of infectious or infusionrelated complications of tocilizumab were low, which is comparable with a previous report [12]. However, 3 patients had a moderately severe reduction in absolute neutrophil count. Therefore, close follow-up with complete blood counts and surveillance for infectious complications is warranted during tocilizumab treatment.

A few limitations of the present study should be addressed. First, this is not a prospective trial. Although clinical and laboratory profiles were comparable among the subgroups, there might still be hidden bias. Second, the observation group had a longer time to first-line immunotherapy and rituximab, and had more frequent $\mathrm{mRS} \leq 2$ at the baseline time point, compared with the other groups. This might be attributable to the retrospective study design and that treatment strategies were decided according to the clinical status of the patient. As the observation group had more frequent mild clinical severity at the baseline time point, physicians might have initially decided to follow-up without immunotherapies for those cases, until those immunotherapies would have been clinically inevitable. Third, there was a lack of standardized treatment and follow-up protocols. Fourth, follow-up time was not very long in this study, considering that clinical improvement was observed after 24 months from onset in NMDAR encephalitis [9]. Fifth, antiglutamic acid decarboxylase 65 antibody, clinical follow-up point. ${ }^{*} p<0.05$ for the tocilizumab group $v s$ the observation group; ${ }^{\dagger} p<0.05$ for the tocilizumab group $v s$ the additional rituximab group; ${ }^{\star} p<0.05$ for the additional rituximab group $v s$ the observation group *Baseline: at the time of tocilizumab initiation in the tocilizumab group, at the time of monthly rituximab therapy initiation in the additional rituximab group, and at 1 month after the last cycle of weekly rituximab therapy in the observation group

known to be associated with various types of $\mathrm{AE}$, was not checked. Additionally, the study was conducted on a relatively small population, which might be a reason for the absence of other significant prognostic factors. Further randomized controlled studies in larger populations are warranted to address these issues.

Acknowledgments This study was supported by grants from the Korean Health Technology R\&D Project (HI13C1558, HI14C25020000) by Ministry of Health \& Welfare, and the Mid-career Researcher Program (NRF-2014R1A2A1A11052709) through the National Research Foundation of Korea (NRF) funded by the Ministry of Science, ICT (Information \& Communication Technology) and future Planning, South Korea. Professor. Kon Chu was supported by grant no. 25-2014-0040 and 03-2015-0430 from the Seoul National University Hospital Research Fund.

Required Author Forms Disclosure forms provided by the authors are available with the online version of this article.

\section{Compliance with Ethical Standards}

Conflicts of interest The authors have no conflicts of interest.

\section{References}

1. Lancaster E, Martinez-Hernandez E, Dalmau J. Encephalitis and antibodies to synaptic and neuronal cell surface proteins. Neurology 2011;77:179-189.

2. Zuliani L, Graus F, Giometto B, Bien C, Vincent A. Central nervous system neuronal surface antibody associated syndromes: review 
and guidelines for recognition. J Neurol Neurosurg Psychiatry 2012:jnnp-2011-301237.

3. Gable MS, Sheriff H, Dalmau J, Tilley DH, Glaser CA. The frequency of autoimmune $\mathrm{N}$-methyl-D-aspartate receptor encephalitis surpasses that of individual viral etiologies in young individuals enrolled in the California Encephalitis Project. Clin Infect Dis 2012;54:899-904.

4. Armangue T, Leypoldt F, Dalmau J. Autoimmune encephalitis as differential diagnosis of infectious encephalitis. Curr Opin Neurol 2014:27:361-368.

5. Graus F, Titulaer MJ, Balu R, et al. A clinical approach to diagnosis of autoimmune encephalitis. Lancet Neurol 2016;15:391-404.

6. Singh TD, Fugate JE, Rabinstein AA. The spectrum of acute encephalitis causes, management, and predictors of outcome. Neurology 2015;84:359-366.

7. Hacohen Y, Wright S, Waters P, et al. Paediatric autoimmune encephalopathies: clinical features, laboratory investigations and outcomes in patients with or without antibodies to known central nervous system autoantigens. J Neurol Neurosurg Psychiatry 2013;84:748-755.

8. Lee W-J, Lee S-T, Byun J-I, et al. Rituximab treatment for autoimmune limbic encephalitis in an institutional cohort. Neurology 2016:10.1212/WNL. 0000000000002635.

9. Titulaer MJ, McCracken L, Gabilondo I, et al. Treatment and prognostic factors for long-term outcome in patients with anti-NMDA receptor encephalitis: an observational cohort study. Lancet Neurol 2013;12:157-165.

10. Kim SJ, Lee JW, Jung CW, et al. Weekly rituximab followed by monthly rituximab treatment for steroid-refractory chronic graftversus-host disease: results from a prospective, multicenter, phase II study. Haematologica 2010;95:1935-1942.

11. Hainsworth JD, Litchy S, Shaffer DW, Lackey VL, Grimaldi M, Greco FA. Maximizing therapeutic benefit of rituximab: maintenance therapy versus re-treatment at progression in patients with indolent non-Hodgkin's lymphoma - a randomized phase II trial of the Minnie Pearl Cancer Research Network. J Clin Oncol 2005;23:1088-1095.

12. Tanaka T, Narazaki M, Kishimoto T. Anti-interleukin-6 receptor antibody, tocilizumab, for the treatment of autoimmune diseases. FEBS Lett 2011;585:3699-3709.

13. Okada M, Kitahara M, Kishimoto S, Matsuda T, Hirano T, Kishimoto T. IL-6/BSF-2 functions as a killer helper factor in the in vitro induction of cytotoxic T cells. J Immunol 1988;141:1543-1549.

14. Kimura A, Kishimoto T. IL-6: Regulator of Treg/Th17 balance. Eur J Immunol 2010;40:1830-1835.

15. Kishimoto T. Interleukin-6: from basic science to medicine-40 years in immunology. Annu Rev Immunol 2005;23:1-21.

16. Smolen JS, Beaulieu A, Rubbert-Roth A, et al. Effect of interleukin6 receptor inhibition with tocilizumab in patients with rheumatoid arthritis (OPTION study): a double-blind, placebo-controlled, randomised trial. Lancet 2008;371:987-997.

17. Nishimoto N, Miyasaka N, Yamamoto K, Kawai S, Takeuchi T, Azuma J. Long-term safety and efficacy of tocilizumab, an anti-IL-6 receptor monoclonal antibody, in monotherapy, in patients with rheumatoid arthritis (the STREAM study): evidence of safety and efficacy in a 5-year extension study. Ann Rheum Dis 2009;68:1580-1584.

18. Illei GG, Shirota Y, Yarboro $\mathrm{CH}$, et al. Tocilizumab in systemic lupus erythematosus: Data on safety, preliminary efficacy, and impact on circulating plasma cells from an open-label phase I dosageescalation study. Arthritis Rheum 2010;62:542-552.
19. Araki M, Matsuoka T, Miyamoto K, et al. Efficacy of the anti-IL-6 receptor antibody tocilizumab in neuromyelitis optica A pilot study. Neurology 2014;82:1302-1306.

20. Ayzenberg I, Kleiter I, Schröder A, et al. Interleukin 6 receptor blockade in patients with neuromyelitis optica nonresponsive to anti-CD20 therapy. JAMA Neurol 2013;70:394-397.

21. Ringelstein M, Ayzenberg I, Harmel J, et al. Long-term Therapy with interleukin 6 receptor blockade in highly active neuromyelitis optica spectrum disorder. JAMA Neurol 2015;72:756-763.

22. Krogias C, Hoepner R, Müller A, Schneider-Gold C, Schröder A, Gold R. Successful treatment of anti-Caspr2 syndrome by interleukin 6 receptor blockade through tocilizumab. JAMA Neurol 2013;70:1056-1059.

23. Irani SR, Vincent A. Targeting the interleukin 6 receptor to treat neuromyelitis optica. JAMA Neurol 2015;72:747-748.

24. Venkatesan A, Tunkel A, Bloch K, et al. Case definitions, diagnostic algorithms, and priorities in encephalitis: consensus statement of the international encephalitis consortium. Clin Infect Dis 2013;57: 1114-1128.

25. Kim T-J, Lee S-T, Shin J-W, et al. Clinical manifestations and outcomes of the treatment of patients with GABA B encephalitis. J Neuroimmunol 2014;270:45-50.

26. Lim J-A, Lee S-T, Jung K-H, et al. Anti-N-methyl-d-aspartate receptor encephalitis in Korea: clinical features, treatment, and outcome. J Clin Neurol 2014;10:157-161.

27. Sunwoo J-S, Lee S-T, Byun J-I, et al. Clinical manifestations of patients with CASPR2 antibodies. J Neuroimmunol 2015;281:17-22.

28. Tunkel AR, Glaser CA, Bloch KC, et al. The management of encephalitis: clinical practice guidelines by the Infectious Diseases Society of America. Clin Infect Dis 2008;47:303-327.

29. Gürcan HM, Keskin DB, Stern JN, Nitzberg MA, Shekhani H, Ahmed AR. A review of the current use of rituximab in autoimmune diseases. Int Immunopharmacol 2009;9:10-25.

30. Common Terminology Criteria for Adverse Events (CTCAE v4.0). Available at: http://evs.nci.nih.gov/ftp1/CTCAE/CTCAE_403 2010-06-14 QuickReference 5x7.pdf. Accessed April 1, 2015.

31. Dale RC, Brilot F, Duffy LV, et al. Utility and safety of rituximab in pediatric autoimmune and inflammatory CNS disease. Neurology 2014;83:142-150

32. Ogura H, Murakami M, Okuyama Y, et al. Interleukin-17 promotes autoimmunity by triggering a positive-feedback loop via interleukin-6 induction. Immunity 2008;29:628-636.

33. Gijbels K, van Damme J, Proost P, Put W, Carton H, Billiau A. Interleukin 6 production in the central nervous system during experimental autoimmune encephalomyelitis. Eur J Immunol 1990;20:233-235.

34. Gruol D, Nelson T. Physiological and pathological roles of interleukin-6 in the central nervous system. Mol Neurobiol 1997:15:307-339.

35. Irani SR, Gelfand JM, Al-Diwani A, Vincent A. Cell-surface central nervous system autoantibodies: Clinical relevance and emerging paradigms. Ann Neurol 2014;76:168-184.

36. McLaughlin P, Grillo-López AJ, Link BK, et al. Rituximab chimeric anti-CD20 monoclonal antibody therapy for relapsed indolent lymphoma: half of patients respond to a four-dose treatment program. J Clin Oncol 1998;16:2825-2833.

37. Wingerchuk DM, Lennon VA, Lucchinetti CF, Pittock SJ, Weinshenker BG. The spectrum of neuromyelitis optica. Lancet Neurol 2007;6:805-815. 